\title{
Exact Solutions For some nonlinear Partial Differential Equations by A Variation of $\left(\mathbf{G}^{\prime} / \mathbf{G}\right)$-Expansion Method:
}

\author{
A. R. Shehata ${ }^{1^{*}}$, F. Abdel Basser ${ }^{1}$, Safaa S. M. abu-amra ${ }^{1,2}$ \\ ${ }^{1}$ Department of Mathematics, Faculty of Science, Minia University, 61519 Minia, Egypt \\ ${ }^{2}$ Department of Mathematics Faculty of Science, Omar Al-Mukhtar University, Al-Bayda. Libya \\ *Correspondence: shehata1433@yahoo.com; Tel: +20 862092239; Fax: +20 862363011
}

\section{Article information}

Received: 10 April 2019

Revised: 26 June 2019

Accepted: 26 June 2019

\section{Key words}

The variation of $\left(G^{\prime} / G\right)$-expansion method some nonlinear partial differential equations Exact solutions.

\begin{abstract}
In this article, we study exact solutions of nonlinear of Burger's Equation, Symmetric Regularized Long Wave (SRLW) equation, and Whitham-BroerKaup equations by using the variation of $\left(\mathrm{G}^{\prime} / \mathrm{G}\right)$-expansion method, respectively. With the aid of mathematical software Maple, we can obtain the exact solutions for the above equations. Here we use the variation of $\left(\mathrm{G}^{\prime} / \mathrm{G}\right)$-expansion method by applying it to solve the above mentioned equations, some new exact traveling wave solutions are obtained which include solitary wave solutions. When the arbitrary constants are taken some special values, the periodic and soliton solutions are obtained from the travelling wave solutions. The obtained solutions are new and not found elsewhere. It is shown that the methods are effective and can be used for many other nonlinear evolution equations (NLEEs) in mathematical physics. This methods is effectual, uncomplicated and can also be used to tackle a number of other differential equations related to mathematical physics
\end{abstract}

\section{Introduction}

As we know, many phenomena's of physics and biology can be represented by Nonlinear PDEs [1-6]. We extended the known $\left(\boldsymbol{G}^{\prime} / \boldsymbol{G}\right)$-expansion method [7-9] to it, then we find here its exact solutions.

A. R. Shehata, used the modified $\left(G^{\prime} / G\right)$-expansion method [10], $(w / g)$-expansion method [11], Geometrical Properties and Exact Solutions of Three $(3+1)$ - Dimensional Nonlinear Evolution Equations in Mathematical Physics Using Different Expansion Methods [12].

Here we use the variation of $\left(\boldsymbol{G}^{\prime} / \boldsymbol{G}\right)$-expansion method by applying it to solve the above mentioned equations; some new exact traveling wave solutions are obtained which include solitary wave solutions. It is shown that the methods are effective and can be used for many other nonlinear evolution equations (NLEEs) in mathematical physics.

\section{Description of the variation of $\left(G^{\prime} / G\right)$-expansion method}

We use the variation of $\left(G^{\prime} / G\right)$-expansion method $[13,14]$ to solve the above equations.

Let

$P\left(u, u_{t}, u_{x}, u_{y}, u_{t t}, u_{t x}, u_{x x}, u_{x y}, u_{y y}, \ldots\right)=0$,

Where $P$ is a polynomial of $u$ and its partial derivatives.

Step 1. Use the solitary wave variable and transformation

$\boldsymbol{u}(\boldsymbol{x}, \boldsymbol{y}, \boldsymbol{t})=\boldsymbol{U}(\xi)$,

$\xi=\tau x+\delta y+\lambda t$ where $\boldsymbol{\tau}, \boldsymbol{\delta}$ and $\boldsymbol{\lambda}$ are nonzero arbitrary constants, we can rewrite Eq.(2.1) as the following nonlinear ODE:

$\boldsymbol{Q}\left(\boldsymbol{U}, \boldsymbol{U}^{\prime}, \boldsymbol{U}^{\prime \prime}, \boldsymbol{U}^{\prime \prime \prime}, \ldots\right)=\mathbf{0}, \quad$ where $\left(^{\prime}\right)=\frac{d}{d \xi}$.

Step2. Suppose

$\boldsymbol{u}(\xi)=\sum_{i=1}^{m} \boldsymbol{a}_{i}\left(G^{\prime} / G\right)^{i}+\sum_{i=1}^{m} b_{i}\left(G^{\prime} / G\right)^{i-1}\left(F^{\prime} / F\right)$,

where $\boldsymbol{G}=\boldsymbol{G}(\xi)$ and $\boldsymbol{F}=\boldsymbol{F}(\xi)$ expresses the solution of the coupled Riccati equation,

$\boldsymbol{G}^{\prime}(\xi)=-\boldsymbol{G}(\xi) . \boldsymbol{F}(\xi)$,

$F^{\prime}(\xi)=1-F(\xi)^{2}$,

$\boldsymbol{a}_{\boldsymbol{i}}(\boldsymbol{i}=\mathbf{0}, \mathbf{1}, \ldots, \boldsymbol{m}), \boldsymbol{b}_{\boldsymbol{i}}(\boldsymbol{i}=\mathbf{1}, \mathbf{2}, \ldots, \boldsymbol{m})$ are constants to be determined later.

These governing equations lead us two types of general solutions:

$G(\xi)= \pm \operatorname{sech}(\xi), F(\xi)=\tanh (\xi)$,
$G(\xi)= \pm \operatorname{csch}(\xi), F(\xi)=\operatorname{coth}(\xi)$.

Step 3. By considering the homogeneous balance between the highest order derivatives and the nonlinear terms appearing in Eq. (2.4), we can find the positive integer $\boldsymbol{m}$ as follows:

$\boldsymbol{D}\left[\boldsymbol{U}^{r}\left(\frac{\boldsymbol{d}^{q} U}{\boldsymbol{d} \xi^{q}}\right)^{\boldsymbol{s}}\right]=\boldsymbol{m} \boldsymbol{r}+\boldsymbol{s}(\boldsymbol{q}+\boldsymbol{m})$, where $D$ denotes the degree of the expression .

Step 4. Substituting Eq.(2.5) into Eq.(2.4) and using Eq.(2.6) and Eq.(2.7), collecting all terms with the same order of $\left(\boldsymbol{G}^{\prime} / \boldsymbol{G}\right)$ or $(\boldsymbol{F})$ together, left-hand side of Eq.(2.4) is converted into another polynomial in $\left(\boldsymbol{G}^{\prime} / \boldsymbol{G}\right)$ 
or $(\boldsymbol{F})$. Equating each coefficient of this polynomial to zero, yields a set of algebraic equations for $\boldsymbol{a}_{\boldsymbol{i}}, \boldsymbol{b}_{\boldsymbol{i}}, \boldsymbol{r}_{\boldsymbol{i}}$ and $\tau, \boldsymbol{\delta}$ and $\boldsymbol{\lambda}$.

Step 5. Determining the constants $\boldsymbol{a}_{\boldsymbol{i}}, \boldsymbol{b}_{\boldsymbol{i}}, \boldsymbol{r}_{\boldsymbol{i}}$ and $\boldsymbol{\tau}, \boldsymbol{\delta}$ and $\lambda$. by solving the algebraic equations in step 5 . As the general solutions of Eq.(2.6) and Eq.(2.7) are already known to us ,then substituting $\boldsymbol{a}_{\boldsymbol{i}}, \boldsymbol{b}_{\boldsymbol{i}}, \boldsymbol{r}_{\boldsymbol{i}}, \boldsymbol{\tau}, \boldsymbol{\delta}, \boldsymbol{\lambda}$ and the general solutions of Eq.(2.6) and Eq.(2.7), we obtain the exact solutions of Eq.(2.1).

\section{Applications of the method:}

\subsection{Example 1: The Burgers' Equation [15]:}

$u_{t}+a u u_{x}+b u_{x x}=0$

It can be observed that the transform:

$\boldsymbol{u}(\boldsymbol{x}, \boldsymbol{t})=U(\xi), \quad \xi=\tau x+\lambda t$

where $\tau$ and $\lambda$ are constants, permits us to reduce the Equation (3.1.1) into an ODE. After integrating once, we have the following form:

$C+\lambda U+\frac{a}{2} \tau U^{2}+b \tau^{2} U^{\prime}=\mathbf{0}$,

where $\boldsymbol{C}$ is a constant of integration. Now by considering the homogeneous balance between the order of $\boldsymbol{U}^{\prime}$ and $\boldsymbol{U}^{2}$ in Eq.(3.1.3), we obtain $\boldsymbol{m}=\mathbf{1}$.

By using step 2 the solution of Eq. (3.1.3), can be written as,

$\begin{aligned} U(\xi) & =a_{0}+a_{1}\left(G^{\prime} / G\right)+b_{1}\left(F^{\prime} / F\right) \\ & =a_{0}-a_{1} F+b_{1}\left(F^{-1}-F\right),\end{aligned}$

Substituting Eq. (3.1.4) into Eq. (3.1.3), collecting the coefficients of $(\boldsymbol{F})^{i}(\boldsymbol{i}=\mathbf{0}, \pm \mathbf{1}, \pm \mathbf{2})$, and letting it be zero, yields a set of simultaneous algebraic equations for $\boldsymbol{a}_{\mathbf{0}}, \boldsymbol{a}_{\mathbf{1}}, \boldsymbol{b}_{\mathbf{1}}, \boldsymbol{\tau}$ and $\boldsymbol{\lambda}$ as follows:

$F^{0}: C+\lambda a_{0}+\frac{1}{2} \tau a a_{0}{ }^{2}-\tau a a_{1} b_{1}-\tau a b_{1}{ }^{2}-\tau^{2} b a_{1}=0$,

$F^{1}:-a a_{0} a_{1} \tau-a a_{0} b_{1} \tau-\lambda a_{1}-\lambda b_{1}=0$,

$F^{2}: \frac{1}{2} \tau a a_{1}{ }^{2}+\tau a a_{1} b_{1}+\frac{1}{2} \tau a b_{1}{ }^{2}+\tau^{2} b a_{1}+\tau^{2} b b_{1}=0$,

$F^{-1}: a a_{0} b_{1} \tau+\lambda b_{1}=0$,

$F^{-2}: \frac{1}{2} \tau a b_{1}^{2}-\tau^{2} b b_{1}=0$.

After solving these algebraic equations for $\boldsymbol{a}_{0}, \boldsymbol{a}_{1}, \boldsymbol{b}_{1}, \boldsymbol{\tau}$ and $\boldsymbol{\lambda}$ with the help of software Maple, yields the following results.

Case 1:

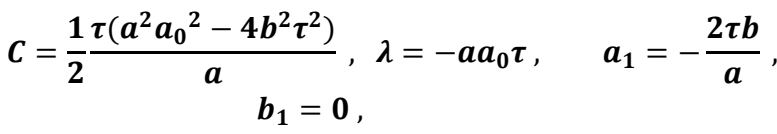

where $\boldsymbol{a}_{\mathbf{0}}, \boldsymbol{\tau}$ are arbitrary.

Case 2:

$C=\frac{1}{4} \frac{a^{2} b_{1}\left(a_{0}{ }^{2}-b_{1}{ }^{2}\right)}{b}, \lambda=-\frac{1}{2} \frac{a^{2} a_{0} b_{1}}{b}, a_{1}=-b_{1}, \tau=$

$\frac{1}{2} \frac{a b_{1}}{b}$

where $\boldsymbol{b}_{1}, \boldsymbol{a}_{\mathbf{0}}$ is arbitrary.
Substituting Eqs. (3.1.10), (3.1.11) into Eq.(3.1.4) we get two types of the exact solutions of Eq.(3.1.1) as follows:

According to case 1 .

Type 1:

Class I: $\quad U_{11}(x, t)=a_{0}+\frac{2 \tau b}{a} \tanh \left(\tau x-a a_{0} \tau t\right)$.

Class II: $\quad U_{12}(x, t)=a_{0}+\frac{2 \tau b}{a} \operatorname{coth}\left(\tau x-a a_{0} \tau t\right)$.

According to case 2 .

Type 2:

Class I: $\quad U_{21}(x, t)=a_{0}+b_{1} \operatorname{coth}\left(\frac{a b_{1}}{2 b} x-\frac{a^{2} a_{0} b_{1}}{2 b} t\right)$.

Class II: $\quad U_{22}(x, t)=a_{0}+b_{1} \tanh \left(\frac{a b_{1}}{2 b} x-\frac{a^{2} a_{0} b_{1}}{2 b} t\right)$.

\subsection{Example 2: The SRLW equation:}

$\boldsymbol{u}_{t t}+\boldsymbol{u}_{x x}+\boldsymbol{u} \boldsymbol{u}_{x t}+\boldsymbol{u}_{x} \boldsymbol{u}_{t}+\boldsymbol{u}_{x x t t}=0$,

which arises in several physical applications including ion sound waves in plasma. It arises in many nonlinear problems of mathematical physics and applied mathematics. Periodic wave solutions of SRLW have been given by using the Exp function method [16], and $\left(G^{\prime} / G\right)$-expansion method [17].

It can be observed that the transform:

$\boldsymbol{u}(\boldsymbol{x}, \boldsymbol{t})=\boldsymbol{U}(\xi), \quad \xi=\tau x+\lambda t$,

where $\boldsymbol{\tau}$ and $\lambda$ are constants, permits to reduce the Equation (3.2.1) into an ODE. After integrating twince, we have the following form:

$\left(\lambda^{2}+\tau^{2}\right) U+\frac{1}{2} \lambda \tau U^{2}+\lambda^{2} \tau^{2} U^{\prime \prime}+C=0$,

Balancing the order of $\boldsymbol{U}^{\prime \prime}$ and $\boldsymbol{U}^{2}$ in Eq.(3.2.3), we obtain $\boldsymbol{m}=\mathbf{2}$.

By using step 2 the solution of Eq. (3.2.3), can be written as,

$$
\begin{aligned}
U(\xi)= & a_{0}+a_{1}\left(G^{\prime} / G\right)+a_{2}\left(G^{\prime} / G\right)^{2}+b_{1}\left(F^{\prime} / F\right) \\
& +b_{2}\left(G^{\prime} / G\right)\left(F^{\prime} / F\right) \\
= & a_{0}-a_{1} F+a_{2} F^{2}+b_{1}\left(F^{-1}-F\right)-b_{2}\left(1-F^{2}\right)
\end{aligned}
$$

Substituting Eq. (3.2.4) into Eq. (3.2.3), collecting the coefficients of $(F)^{i}(i=0, \pm 1, \pm 2, \pm 3, \pm 4)$, and letting it be zero, yields a set of simultaneous algebraic equations for $\boldsymbol{a}_{0}, \boldsymbol{a}_{1}, \boldsymbol{a}_{2}, \boldsymbol{b}_{1}, \boldsymbol{b}_{2}, \boldsymbol{\tau}$ and $\boldsymbol{\lambda}$ as follows:

$$
\begin{gathered}
F^{4}: \frac{1}{2} \tau \lambda a_{2}{ }^{2}+\frac{1}{2} \tau \lambda b_{2}{ }^{2}+6 \tau^{2} \lambda^{2} a_{2}+6 \tau^{2} \lambda^{2} b_{2} \\
+\tau \lambda a_{2} b_{2}=0, \\
F^{3}:-2 \tau^{2} \lambda^{2} a_{1}-2 \tau^{2} \lambda^{2} b_{1}-\tau \lambda a_{1} a_{2}-\tau \lambda a_{1} b_{2} \\
-\tau \lambda a_{2} b_{1}-\tau \lambda b_{1} b_{2}=0, \\
F^{2}: \frac{1}{2} \tau \lambda a_{1}{ }^{2}+\frac{1}{2} \tau \lambda b_{1}{ }^{2}-\tau \lambda b_{2}{ }^{2}-8 \tau^{2} \lambda^{2} a_{2}- \\
8 \tau{ }^{2} \lambda^{2} b_{2}+\tau \lambda a_{1} b_{1}+\tau \lambda a_{0} b_{2}+\tau \lambda a_{0} a_{2}- \\
\tau \lambda a_{2} b_{2}+a_{2} \tau^{2}+b_{2} \lambda^{2}+b_{2} \tau^{2}+a_{2} \lambda^{2}=0, \\
F^{1}: 2 \tau^{2} \lambda^{2} a_{1}+2 \tau^{2} \lambda^{2} b_{1}-\tau \lambda a_{0} a_{1}-\tau \lambda a_{0} b_{1}+ \\
\tau \lambda a_{1} b_{2}+\tau \lambda a_{2} b_{1}+2 \tau \lambda b_{1} b_{2}-\tau a_{1}-\tau^{2} b_{1}- \\
\lambda^{2} a_{1}-\lambda^{2} b_{1}=0,
\end{gathered}
$$




$$
\begin{aligned}
& F^{0}:-\tau \lambda a_{1} b_{1}-\tau \lambda a_{0} b_{2}+C+\frac{1}{2} \tau \lambda b_{2}{ }^{2}-\tau \lambda b_{1}{ }^{2}+ \\
& 2 \tau^{2} \lambda^{2} a_{2}+2 \tau^{2} \lambda^{2} b_{2}+\frac{1}{2} \tau \lambda a_{0}^{2}+a_{0} \tau^{2}+a_{0} \lambda^{2}- \\
& b_{2} \tau^{2}-b_{2} \lambda^{2}=0, \\
& F^{-1}:-2 \tau^{2} \lambda^{2} b_{1}+\tau \lambda a_{0} b_{1}-\tau \lambda b_{1} b_{2}+\tau^{2} b_{1}+\lambda^{2} b_{1}= \\
& 0, \\
& F^{-2}: \frac{1}{2} \tau \lambda b_{1}^{2}=0, \\
& F^{-3}: 2 \tau^{2} \lambda^{2} b_{1}=0 .
\end{aligned}
$$

After solving these algebraic equations for $\boldsymbol{a}_{0}, \boldsymbol{a}_{1}, \boldsymbol{a}_{2}, \boldsymbol{b}_{1}, \boldsymbol{b}_{2}, \boldsymbol{\tau}, \boldsymbol{\lambda}$ and $\boldsymbol{C}$ with the help of software Maple, yields the following results.

\section{Case 1:}

$C=-\frac{1}{2} \tau \lambda a_{0}{ }^{2}+\tau \lambda a_{0} b_{2}-\frac{1}{2} \tau \lambda{b_{2}}^{2}-a_{0} \tau^{2}+b_{2} \tau^{2}-$ $a_{0} \lambda^{2}-b_{2} \lambda^{2}, a_{1}=0, a_{2}=-b_{2}, b_{1}=0$,

where $\boldsymbol{\tau}, \boldsymbol{\lambda}, \boldsymbol{a}_{\mathbf{0}}, \boldsymbol{b}_{\mathbf{2}}$ are arbitrary.

Case 2:

$$
\begin{aligned}
C=-\frac{1}{2} \frac{16 \tau^{4} \lambda^{4}-\tau^{4}-2 \tau^{2} \lambda^{2}-\lambda^{4}}{\tau \lambda}, \\
a_{0}=\frac{8 \tau^{2} \lambda^{2}+\tau \lambda b_{2}-\tau^{2}-\lambda^{2}}{\tau \lambda}, a_{1} \\
=0, a_{2}=-12 \tau \lambda-b_{2}, \quad b_{1}=0,
\end{aligned}
$$

where $\boldsymbol{\tau}, \boldsymbol{\lambda}, \boldsymbol{b}_{\mathbf{2}}$ are arbitrary.

Substituting Eqs.(3.2.13),(3.2.14) into Eq.(3.2.4) we get two types of the exact solutions of Eq.(3.2.1) as follows:

According to case 1.

Type 1:

Class I: $\quad U_{11}(x, t)=a_{0}-b_{2}$.

Class II: $\quad U_{12}(x, t)=a_{0}-b_{2}$.

According to case 2 .

Type 2:

Class I:

$$
U_{21}=8 \tau \lambda-\frac{\tau}{\lambda}-\frac{\lambda}{\tau}-12 \tau \lambda \tanh ^{2}(\tau x+\lambda t) .
$$

Class II:

$$
U_{22}(x, t)=8 \tau \lambda-\frac{\tau}{\lambda}-\frac{\lambda}{\tau}-12 \tau \lambda \operatorname{coth}^{2}(\tau x+\lambda t) .
$$

\subsection{Example 3: The Whitham-Broer-Kaup equations} $[18,19]$ :

$$
\left\{\begin{array}{c}
u_{t}+u u_{x}+v_{x}+p u_{x x}=0 \\
v_{t}+(u v)_{x}-p v_{x x}+q u_{x x x}=0
\end{array}\right.
$$

where $\boldsymbol{u}=\boldsymbol{u}(\boldsymbol{x}, \boldsymbol{t})$ is the field of horizontal velocity, $\boldsymbol{v}=\boldsymbol{v}(\boldsymbol{x}, \boldsymbol{t})$ is the height deviating from the equilibrium position of liquid, $\boldsymbol{p}$ and $\boldsymbol{q}$ are real constants that represent different diffusion powers.

For our purpose, we use the following transformation:

$\{\boldsymbol{u}(\boldsymbol{x}, \boldsymbol{t})=\boldsymbol{U}(\xi), \quad \xi=\tau x-\lambda \boldsymbol{t}$,

$\{v(x, t)=V(\xi), \quad \xi=\tau x-\lambda t$,

where $\lambda \neq 0$ and $\boldsymbol{\tau} \neq \mathbf{0}$ constants. Then by using Eq.(3.3.2), Eq.(3.3.1) can be turned into an ODE: $\left\{\begin{array}{c}-\lambda U^{\prime}+\tau U U^{\prime}+\tau V^{\prime}+\tau^{2} p U^{\prime \prime}=0 \\ -\lambda V^{\prime}+\tau(U V)^{\prime}-\tau^{2} p V^{\prime \prime}+\tau^{3} q U^{\prime \prime \prime}=0\end{array}\right.$

where $\boldsymbol{U}^{\prime}=\boldsymbol{d} \boldsymbol{U} / \boldsymbol{d} \boldsymbol{\xi}$.By integrating once and setting the constants of integration to be zero, we obtain

$\left\{\begin{array}{c}-\lambda U+\tau \frac{U^{2}}{2}+\tau V+\tau^{2} p U^{\prime}=0, \\ -\lambda V+\tau U V-\tau^{2} p V^{\prime}+\tau^{3} q U^{\prime \prime}=0,\end{array}\right.$

balancing the order of $\boldsymbol{U}^{\mathbf{2}}$ and $\boldsymbol{U}^{\prime}, \boldsymbol{U} \boldsymbol{V}$ and $\boldsymbol{U}^{\prime \prime}$ in

Eq.(3.3.4),we obtain:

$$
m=1, n=2 .
$$

By using step 2 the solutions of Eqs. (3.3.4) can be written as,

$$
\begin{aligned}
U(\xi) & =a_{0}+a_{1}\left(\frac{G \prime}{G}\right)+b_{1}\left(\frac{F^{\prime}}{F}\right) \\
& =a_{0}-a_{1} F+b_{1}\left(F^{-1}-F\right) \\
V(\xi) & =c_{0}+c_{1}\left(\frac{G \prime}{G}\right)+c_{2}\left(\frac{G^{\prime}}{G}\right)^{2}+d_{1}\left(\frac{F^{\prime}}{F}\right)+d_{2}\left(\frac{G \prime}{G}\right)\left(\frac{F \prime}{F}\right), \\
& =c_{0}-c_{1} F+c_{2} F^{2}+d_{1}\left(F^{-1}-F\right)-d_{2}\left(1-F^{2}\right),
\end{aligned}
$$

substituting Eqs. (3.3.6),(3.3.7) into Eqs.(3.3.4), collecting the coefficients of $(F)^{i}(i=0, \pm 1, \pm 2, \pm 3)$, and letting it be zero, yields a set of simultaneous algebraic equations for $\boldsymbol{a}_{0}, \boldsymbol{a}_{1}, \boldsymbol{b}_{1}, \boldsymbol{c}_{0}, \boldsymbol{c}_{1}, \boldsymbol{c}_{2}, \boldsymbol{d}_{1}, \boldsymbol{d}_{2}, \boldsymbol{\tau}$ and $\lambda$ as follows:

$$
\begin{aligned}
& F^{0}:-\lambda a_{0}+\frac{1}{2} \tau a_{0}{ }^{2}-\tau b_{1}{ }^{2}+\tau c_{0}-\tau d_{2}-\tau a_{1} b_{1}-\tau^{2} p a_{1} \\
& =0 \\
& F^{1}:-a_{0} a_{1} \tau-a_{0} b_{1} \tau+a_{1} \lambda+b_{1} \lambda-c_{1} \tau-d_{1} \tau=0 \\
& F^{2}: \tau a_{1} b_{1}+\tau^{2} p a_{1}+\tau^{2} p b_{1}+\tau d_{2}+\frac{1}{2} \tau b_{1}{ }^{2}+\tau c_{2} \\
& +\frac{1}{2} \tau a_{1}^{2}=0
\end{aligned}
$$

$F^{-1}: a_{0} b_{1} \tau-b_{1} \lambda+d_{1} \tau=0$

$F^{-2}:-\tau^{2} p b_{1}+\frac{1}{2} \tau b_{1}{ }^{2}=0$,

$F^{0}: \tau^{2} p c_{1}+\tau a_{0} c_{0}-\tau a_{0} d_{2}-\tau a_{1} d_{1}-\tau b_{1} c_{1}-2 \tau b_{1} d_{1}-$ $\lambda c_{0}+\lambda d_{2}=0$,

$F^{1}: 2 a_{1} \tau^{3} q+2 b_{1} \tau^{3} q-2 c_{2} \tau^{2} p-2 d_{2} \tau^{2} p-a_{0} c_{1} \tau-$ $a_{0} d_{1} \tau-a_{1} c_{0} \tau+a_{1} d_{2} \tau-b_{1} c_{0} \tau+b_{1} c_{2} \tau+$ $2 b_{1} d_{2} \tau+\lambda c_{1}+\lambda d_{1}=0$,

$$
\begin{array}{r}
F^{2}:-c_{1} \tau^{2} p-d_{1} \tau^{2} p+a_{0} c_{2} \tau+a_{0} d_{2} \tau+a_{1} c_{1} \tau+a_{1} d_{1} \tau \\
+b_{1} c_{1} \tau+b_{1} d_{1} \tau-\lambda c_{2}-\lambda d_{2}=0
\end{array}
$$

$F^{3}:-2 a_{1} \tau^{3} q-2 b_{1} \tau^{3} q+2 c_{2} \tau^{2} p+2 d_{2} \tau^{2} p-a_{1} c_{2} \tau-$ $a_{1} d_{2} \tau-b_{1} c_{2} \tau-b_{1} d_{2} \tau=0$,

$F^{-1}:-2 b_{1} \tau^{3} q+a_{0} d_{1} \tau+b_{1} c_{0} \tau-b_{1} d_{2} \tau-\lambda d_{1}=0$,

$F^{-2}: d_{1} \tau^{2} p+b_{1} d_{2} \tau=0$,

$F^{-3}: 2 \tau^{3} q b_{1}=0$.

After solving these algebraic equations for $\boldsymbol{a}_{0}, \boldsymbol{a}_{1}, \boldsymbol{b}_{1}, \boldsymbol{c}_{\mathbf{0}}, \boldsymbol{c}_{1}, \boldsymbol{c}_{2}, \boldsymbol{d}_{1}, \boldsymbol{d}_{2}, \boldsymbol{\tau}$ and $\lambda$ with the help of software Maple, yields the following results.

Case 1: 


$$
\begin{aligned}
a_{0}=\frac{\lambda}{\tau}, a_{1}=-\frac{\lambda}{\tau} & , b_{1}=0, c_{0} \\
& =\frac{1}{2} \frac{-2 \lambda \tau^{2} p+2 d_{2} \tau^{2}+\lambda^{2}}{\tau^{2}}, c_{1}=0, c_{2} \\
& =-\frac{1}{2} \frac{-2 \lambda \tau^{2} p+2 d_{2} \tau^{2}+\lambda^{2}}{\tau^{2}}, d_{1} \\
& =0, \\
q=\frac{1}{4} \frac{-4 \tau^{4} p^{2}+\lambda^{2}}{\tau^{4}}, &
\end{aligned}
$$

where $\boldsymbol{\lambda}, \boldsymbol{\tau}, \boldsymbol{d}_{\mathbf{2}}, \boldsymbol{p}$ are arbitrary.

\section{Case 2:}

$a_{0}=\frac{\lambda}{\tau}, a_{1}=0, b_{1}= \pm \frac{\lambda}{\sqrt{2} \tau} i, c_{0}=d_{2}, c_{1}=0, c_{2}=$

$\frac{1}{2} \frac{-2 d_{2} \tau^{2}+\lambda^{2}}{\tau^{2}}, d_{1}=0, p= \pm \frac{\lambda}{2 \sqrt{2} \tau^{2}} i, q=0$,

where $\boldsymbol{\lambda}, \boldsymbol{d}_{\mathbf{2}}, \boldsymbol{\tau}$ are arbitrary.

substituting Eqs.(3.3.20),(3.3.21) into

Eqs.(3.3.6),(3.3.7) we get two types of the exact

solutions of Eq.(3.3.1) as follows:

According to case 1 .

Type 1:

Class I: $\quad U_{11}(x, t)=\frac{\lambda}{\tau}+\frac{\lambda}{\tau} \tanh (\tau x-\lambda t)$,

$V_{11}(x, t)=\left(\frac{-2 \lambda \tau^{2} p+2 d_{2} \tau^{2}+\lambda^{2}}{2 \tau^{2}}-d_{2}\right)\left(1-\tanh ^{2}(\tau x+\lambda t)\right)$.

Class II: $\quad U_{12}(x, t)=\frac{\lambda}{\tau}+\frac{\lambda}{\tau} \operatorname{coth}(\tau x-\lambda t)$,

$V_{12}(x, t)=\left(\frac{-2 \lambda \tau^{2} p+2 d_{2} \tau^{2}+\lambda^{2}}{2 \tau^{2}}-d_{2}\right)\left(1-\operatorname{coth}^{2}(\tau x+\lambda t)\right)$.

According to case 2 .

Type 2:

Class I:

$$
\begin{aligned}
& U_{21}=\frac{\lambda}{\tau} \pm \frac{\lambda}{\sqrt{2} \tau} i(\operatorname{coth}(\tau x-\lambda t)-\tanh (\tau x-\lambda t)), \\
& V_{21}=\left(\frac{-2 d_{2} \tau^{2}+\lambda^{2}}{2 \tau^{2}}+d_{2}\right) \tanh ^{2}(\tau x+\lambda t) .
\end{aligned}
$$

Class II:

$$
\begin{aligned}
& U_{22}=\frac{\lambda}{\tau} \pm \frac{\lambda}{\sqrt{2} \tau} i(\tanh (\tau x-\lambda t)-\operatorname{coth}(\tau x-\lambda t)) \\
& V_{22}=\left(\frac{-2 d_{2} \tau^{2}+\lambda^{2}}{2 \tau^{2}}+d_{2}\right) \operatorname{coth}^{2}(\tau x+\lambda t) .
\end{aligned}
$$

\section{Numerical solutions for the exact solutions of the above NPD equations:}

We can study the behavior of the travelling wave solutions which obtained above by illustrating the following figures:

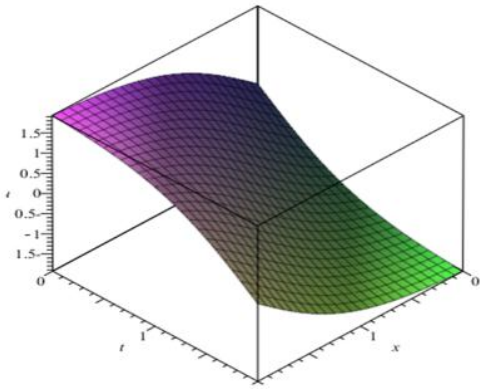

(Figure 1): The exact solution of(3.1.12) When $a_{0}=0, a=b=\tau=1$

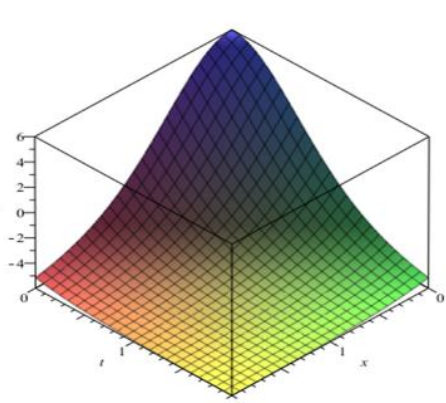

(Figure 3): The exact solution of(3.2.17) When $\lambda=\tau=1$

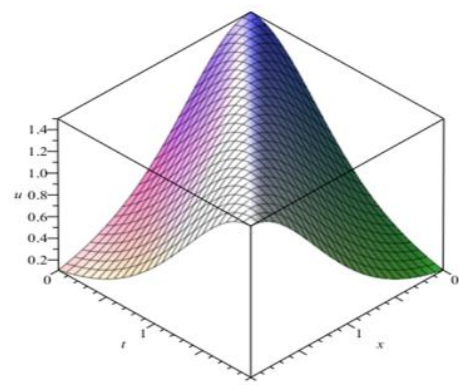

(Figure 5): The exact solution of (3.3.23) When $\lambda=-1, \tau=p=1, d_{2}=0$

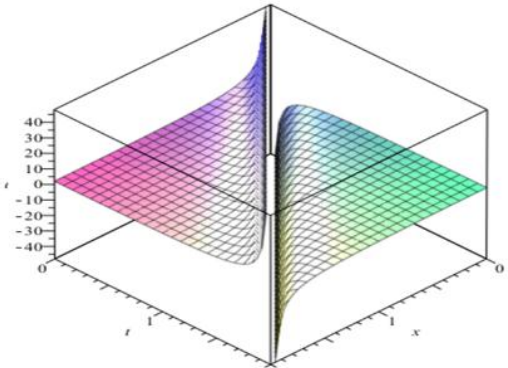

(Figure 2): The exact solution of (3.1.14) When $a_{0}=0, b_{1}=2, a=b=\tau=1$

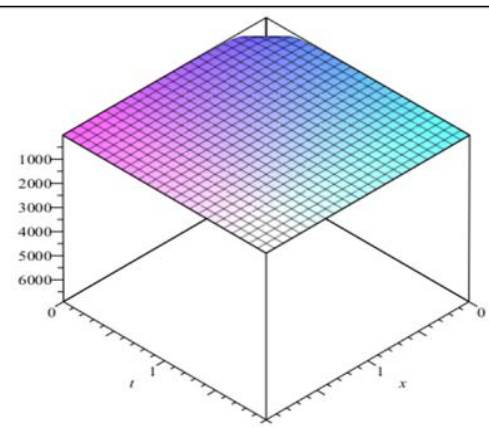

(Figure 4): The exact solution of (3.2.18) When $\lambda=\tau=1$

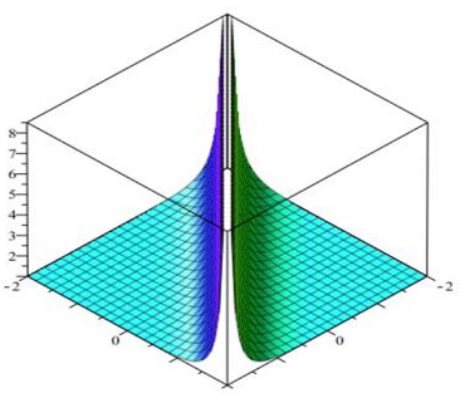

(Figure 6): The exact solution of (3.3.26) When $\lambda=\tau=1$ 


\section{Conclusion}

Here we use the variation of $\left(G^{\prime} / G\right)$-expansion method to solve some NLPDEs, namely nonlinear of Burger's Equation, Symmetric Regularized Long Wave (SRLW) equation and Whitham-Broer-Kaup equations. This method is reliable and efficient and gives new solutions.

And it can be used for many others nonlinear partial differential equations in mathematical physics.

\section{References}

[1] M.J. Ablowitz, P.A. Clarkson, "Solitons, nonlinear evolution equation and inverse scattering," Cambridge University Press, New York (1991).

[2] E.G. Fan, ' Extended tanh-function method and its applications to nonlinear equations", Phys. Lett.A 277 (2000)212-218.

[3] J.H. He, X.H. Wu, ''Exp-function method for nonlinear wave equations, Chaos, solitons and Fractals 30 (2006)700-708.

[4] A.J.M. Jawad, M.D. Petkovic, A. Biswas, "Modified simple equation method for nonlinear evolution equations", Appl. Math. Comput. 217 (2010)869-877.

[5] H. Kheiri, M.R. Moghaddam, V. Vafael, "Applications of the $\left(G^{\prime} / G\right)$ expansion method for the Burgers, Burgers-Huxley and modified Burgers-KdV equations", Pramana J.Phys. 76 (2011)831-842.

[6] M.J. Ablowitz, P.A. Clarkson, "Solitons, Nonlinear Evolution Equations and Inverse Scattering Transform', Cambridge University Press, Cambridge, $U K(1991)$.

[7] M. L. Wang, J. L. Zhang and X. Z. Li, "Application of the $\left(\boldsymbol{G}^{\prime} / \boldsymbol{G}\right)$ expansion to travelling wave solutions of the Broer-Kaup and the approximate long water wave equations, "Appl. Math. Comput., 206 (2008)321-326.

[8] I. Aslan, "Discrete exact solutions to some nonlinear differential-difference equations via the $\left(\boldsymbol{G}^{\prime} / \boldsymbol{G}\right)$ - expansion method, "Appl. Math. Comput., 215 (2009)3140-3147.

[9] B. Ayhan and A. Bekir, " the $\left(\boldsymbol{G}^{\prime} / \boldsymbol{G}\right)$-expansion method for the nonlinear lattice equations, "Commun. Nonlinear Sci. Numer. Simulat., 17 (2012)34903498 .

[10] A.R. Shehata, "The travelling wave solutions of the perturbed nonlinear Schrödinger equation and the cubic-quintic Ginzburg Landau equation using the modified $\left(\mathrm{G}^{\prime} / \mathrm{G}\right)$-expansion method" Applied mathematics and computation 217(1) (2010) 1-10.

[11] A.R. Shehata, Safaa. Abu-Amra, "Traveling wave solutions for some nonlinear partial differential equations by using modified $(w / g)$-expansion method". Eur. J. Math. Sci. 4 (2) (2018)35-58.

[12] A.R. Shehata, Safaa. Abu-Amra, "Geometrical Properties and Exact Solutions of Three (3+1)-Dimensional Nonlinear Evolution Equations in Mathematical Physics Using Different Expansion Methods' J. Advances in Math. Comput. Sci. 32(4) (2019)1-19.

[13] A. Das and A. Ganguly, A Variation of $\left(\boldsymbol{G}^{\prime} / \boldsymbol{G}\right)$-expansion method: Travelling Wave Solutions to Nonlinear Equations, Int. J. Nonlnear Sci., 17(3) (2014)268-280.

[14] R.S. Ibrahim, O.H. El-Kalaawy, G.S. Said, A Variation of $\left(\boldsymbol{G}^{\prime} / \boldsymbol{G}\right)$ expansion method and new exact travelling Wave Solutions of nonlinear reaction-diffusion model, Int. J. of Pure and Applied Mathematics. 101(3) (2015)187-211.

[15] Q. Wang, 'Numerical Solutions for Fractional KDV-Burgers Equation by Adomian Decomposition Method, 'Applied Mathematics and Computation, 182(2) (2006)1048-1055.

[16] F. Xu, Application of exp-function method to symmetric regularized long wave (SRLW) equation, Phy. Lett. A, 372 (2008)252-257.

[17] A. Bekir and A. C. Cevikel, New exact travelling wave solutions of nonlinear physical models, Chaos, Solitons Fract. 41 (2009)1733-1739.

[18] A.M. Wazwaz, Multiple soliton solutions for the Whitham-Broer-Kaup model in the shallow water small-amplitude regime, Phys. Scr.,88 (2013).

[19] E. Fan and H. Zhang, Backlund transformation and exact solutions for Whitham-Broer-Kaup equations in shallow water, Appl. Math. Mech., 19 (1998)713-716. 